hep-ph/9903474

FTUV/99-16

IFIC/99-17

UWThPh-1999-18

\title{
Top-Quark Phenomenology in Models with Bilinearly and Spontaneously Broken R-parity
}

\author{
Lluís Navarro ${ }^{1}$, Werner Porod ${ }^{2}$, and José W. F. Valle ${ }^{1}$ \\ ${ }^{1}$ Departament de Física Teòrica-IFIC, CSIC-Univ. de València \\ Burjassot, València 46100, Spain \\ http://neutrinos.uv.es \\ ${ }^{2}$ Insitut für Theoretische Physik, Univ. Wien \\ A-1090 Vienna, Austria
}

\begin{abstract}
We study unconventional decays of the top-quark in the framework of SUSY models with spontaneously broken R-parity. In particular we discuss an effective theory which consists of the MSSM plus bilinearly broken R-parity. We demonstrate that the decay modes $t \rightarrow \tilde{\tau}_{1}^{+} b$ and $t \rightarrow \tau^{+} \tilde{b}_{1}$ can have large branching ratios even in scenarios where the tau-neutrino mass is very small. We show that existing Tevatron data already probe the theoretical parameters, with promising prospects for further improvement at the Run 2 of the Tevatron.
\end{abstract}




\section{Introduction}

Although remarkably successful in the description of the phenomenology of the strong and electroweak interactions, the Standard Model (SM) leaves unanswered some issues such as the hierarchy problem and the unification of the gauge couplings. These have provided strong impetus to the study of supersymmetric extensions [1], in particular those that break R-parity, RPV models, for short [2]. Of these we will focus on the case of bilinear R-Parity Violation, BRPV for short [3, 4, 5, 6]. They are well-motivated theoretically as they arise as effective truncations of models where R-Parity is broken spontaneously [7] through right handed sneutrino vacuum expectation values (VEV) $\left\langle\tilde{\nu}_{j}^{c}\right\rangle=v_{R j} \neq 0$. These models open new possibilities for the study of the unification of the Yukawa couplings [8]. In particular it has been shown that in BRPV models bottom-tau unification may be achieved at any value of $\tan \beta$. These models predict a plethora of novel processes [9] that could reveal the existence of SUSY in a totally different way, not only through the usual missing momentum signature as predicted by the Minimal Supersymmetric Standard Model (MSSM). They provide a very predictive approach to the violation of $\mathrm{R}$-Parity, which renders the systematic study of R-parity violating physics [9] possible. Also, they are more restrictive than trilinear $\mathrm{R}-$ parity violating (TRPV) models, especially in their supergravity formulation with universal soft-breaking terms at the unification scale, as in [3].

Here we will consider the simplest superpotential which violates R-Parity

$$
W_{\not R_{p}}=W_{M S S M}+\epsilon_{i} \widehat{L}_{i} \widehat{H}_{u},
$$

assuming that tri-linear terms are absent or suppressed, as would be the case if their origin is gravitational [10]. The $\epsilon_{i}$ terms also violate lepton number in the $i$ th generation respectively. In models with spontaneously broken $\mathrm{R}$-Parity [7] the $\epsilon_{i}$ parameters are then identified as equal to some Yukawa coupling times $v_{R j}$. This provides the main theoretical motivation for adding explicitly BRPV to the MSSM superpotential.

It has often been claimed that the BRPV term can be rotated away from the superpotential by a suitable choice of the basis [11]. If this were true the $\epsilon_{i}$ terms would be unphysical. However, one can show that, even though performing this rotation of the superfields indeed eliminates the BRPV, RPV terms are reintroduced in the form of TRPV. Moreover, supersymmetry must be broken and the presence of the $\epsilon_{i}$ terms in the superpotential also introduces R-parity violating terms $\varepsilon_{a b}\left(B_{i} \epsilon_{i} L_{i}^{a} H_{u}^{b}\right)$ in the scalar sector, implying that the vacuum expectation value $\left\langle\tilde{\nu}_{l i}\right\rangle=v_{i} / \sqrt{2}$ is non-zero. This in turn generates more $\mathrm{R}$-parity and lepton number violating terms inducing a tau-neutrino mass. Thus it is in general impossible to rotate away the bilinear term in the Superpotential and in the soft SUSY breaking potential at the same time.

In this model the top-quark gets additional decay modes, e.g. $t \rightarrow \tilde{\tau}_{1}^{+} b$. We study these decays in view of the Tevatron (top decays in TRPV models have been treated in [12]) and show that existing Tevatron data already pose restrictions on the parameter space. This work is organized in the following way: in Sect. 2 we discuss the model working out the necessary details for the discussion of the top decays which will be discussed in Sect. 3. In Sect. 4 we draw our conclusions. 


\section{The model}

For simplicity we set from now on $\epsilon_{1}=\epsilon_{2}=0$, and in this way, only tau-lepton number is violated. In this case, considering only the third generation, the BRPV superpotential has the form:

$$
W_{R_{p}}=\varepsilon_{a b}\left[h_{t} \widehat{Q}_{3}^{a} \widehat{U}_{3} \widehat{H}_{u}^{b}+h_{b} \widehat{Q}_{3}^{b} \widehat{D}_{3} \widehat{H}_{d}^{a}+h_{\tau} \widehat{L}_{3}^{b} \widehat{R}_{3} \widehat{H}_{d}^{a}+\mu \widehat{H}_{u}^{a} \widehat{H}_{d}^{b}+\epsilon_{3} \widehat{L}_{3}^{a} \widehat{H}_{u}^{b}\right],
$$

where the first four terms correspond to the MSSM. The last term violates tau-lepton number and therefore also R-Parity. The soft SUSY breaking potential is given by

$$
V_{\text {soft }}=V_{\text {soft }, M S S M}+B_{2} \epsilon_{3} \widetilde{L}_{3} H_{u}+h . c
$$

where $V_{\text {soft,MSSM }}$ is the usual soft SUSY breaking potential of the MSSM.

The scalar potential contains tadpoles

$$
V_{\text {linear }}=t_{1}^{0} \sigma_{1}^{0}+t_{2}^{0} \sigma_{2}^{0}+t_{3}^{0} \tilde{\nu}_{\tau}^{R}
$$

where

$$
\begin{aligned}
t_{1}^{0} & =\left(m_{H_{d}}^{2}+\mu^{2}\right) v_{d}-B \mu v_{u}-\mu \epsilon_{3} v_{3}+\frac{1}{8}\left(g^{2}+g^{\prime 2}\right) v_{d}\left(v_{d}^{2}-v_{u}^{2}+v_{3}^{2}\right), \\
t_{2}^{0} & =\left(m_{H_{u}}^{2}+\mu^{2}+\epsilon_{3}^{2}\right) v_{u}-B \mu v_{d}+B_{2} \epsilon_{3} v_{3}-\frac{1}{8}\left(g^{2}+g^{\prime 2}\right) v_{u}\left(v_{d}^{2}-v_{u}^{2}+v_{3}^{2}\right), \\
t_{3}^{0} & =\left(m_{L_{3}}^{2}+\epsilon_{3}^{2}\right) v_{3}-\mu \epsilon_{3} v_{d}+B_{2} \epsilon_{3} v_{u}+\frac{1}{8}\left(g^{2}+g^{\prime 2}\right) v_{3}\left(v_{d}^{2}-v_{u}^{2}+v_{3}^{2}\right) .
\end{aligned}
$$

and they are equal to zero at the minimum of the potential. Here $v_{u}, v_{d}$, and $v_{3}$ are the VEVs of $H_{u}^{0}, H_{d}^{0}$, and $\tilde{\nu}_{\tau}$, respectively. $m_{H_{u}}, m_{H_{d}}$, and $m_{L_{3}}$ are the corresponding soft Susy-breaking mass parameters.

The charginos mix with the tau lepton. In a basis where $\psi^{+T}=\left(-i \lambda^{+}, \widetilde{H}_{u}^{+}, \tau_{R}^{+}\right)$ and $\psi^{-T}=\left(-i \lambda^{-}, \widetilde{H}_{d}^{-}, \tau_{L}^{-}\right)$, the charged fermion mass terms in the Lagrangian are

$$
\mathcal{L}_{m}=-\frac{1}{2}\left(\psi^{+T}, \psi^{-T}\right)\left(\begin{array}{cc}
0 & M_{C}^{T} \\
M_{C} & 0
\end{array}\right)\left(\begin{array}{l}
\psi^{+} \\
\psi^{-}
\end{array}\right)+\text {h.c. }
$$

where the chargino/tau mass matrix is given by

$$
M_{C}=\left[\begin{array}{ccc}
M & \frac{1}{\sqrt{2}} g v_{u} & 0 \\
\frac{1}{\sqrt{2}} g v_{d} & \mu & -\frac{1}{\sqrt{2}} h_{\tau} v_{3} \\
\frac{1}{\sqrt{2}} g v_{3} & -\epsilon_{3} & \frac{1}{\sqrt{2}} h_{\tau} v_{d}
\end{array}\right]
$$

and $M$ is the $S U(2)$ gaugino soft mass. Clearly, the chargino sector decouples from the tau sector in the limit $\epsilon_{3}=v_{3}=0$. As in the MSSM, the chargino mass matrix is diagonalized by two rotation matrices $U$ and $V$ defined by

$$
\chi_{i}^{-}=U_{i j} \psi_{j}^{-} \quad ; \quad \chi_{i}^{+}=V_{i j} \psi_{j}^{+}
$$

Then

$$
U^{*} M_{C} V^{-1}=M_{C D}
$$

where $M_{C D}$ is the diagonal charged fermion mass matrix. The tau Yukawa coupling $h_{\tau}$ is chosen such that one of the eigenvalues is equal to the tau mass. This is calculated 
from the vacuum expectation values of the model through an exact tree level relation given by

$$
h_{\tau}^{2}=\frac{2 m_{\tau}^{2}}{v_{d}^{2}}\left(\frac{f+g\left(\varepsilon_{3}, v_{3}\right)}{f-\frac{2}{v_{d}^{2}} h\left(\varepsilon_{3}, v_{3}\right)}\right)
$$

The functions $f, g$ and $h$ are given in ref. [13].

In our model, the tau neutrino acquires mass due to a mixing between the neutralino sector and the tau-neutrino. In the basis $\psi^{0 T}=\left(-i \lambda^{\prime},-i \lambda^{3}, \widetilde{H}_{d}^{1}, \widetilde{H}_{u}^{2}, \nu_{\tau}\right)$ the neutral fermions mass terms in the Lagrangian are given by

$$
\mathcal{L}_{m}=-\frac{1}{2}\left(\psi^{0}\right)^{T} M_{N} \psi^{0}+\text { h.c. }
$$

where

$$
M_{N}=\left[\begin{array}{ccccc}
M^{\prime} & 0 & -\frac{1}{2} g^{\prime} v_{d} & \frac{1}{2} g^{\prime} v_{u} & -\frac{1}{2} g^{\prime} v_{3} \\
0 & M & \frac{1}{2} g v_{d} & -\frac{1}{2} g v_{u} & \frac{1}{2} g v_{3} \\
-\frac{1}{2} g^{\prime} v_{d} & \frac{1}{2} g v_{d} & 0 & -\mu & 0 \\
\frac{1}{2} g^{\prime} v_{u} & -\frac{1}{2} g v_{u} & -\mu & 0 & \epsilon_{3} \\
-\frac{1}{2} g^{\prime} v_{3} & \frac{1}{2} g v_{3} & 0 & \epsilon_{3} & 0
\end{array}\right]
$$

and $M^{\prime}$ is the $U(1)$ gaugino soft mass. This neutralino/neutrino mass matrix is diagonalized by a $5 \times 5$ rotation matrix $N$ such that

$$
N^{*} M_{N} N^{-1}=\operatorname{diag}\left(m_{\chi_{1}^{0}}, m_{\chi_{2}^{0}}, m_{\chi_{3}^{0}}, m_{\chi_{4}^{0}}, m_{\nu_{\tau}}\right)
$$

The physical states $\chi_{j}^{0}$ are given by:

$$
\psi_{j}^{0}=N^{*}{ }_{k j} \chi_{j}^{0} .
$$

There is also a mixing between the charged Higgs boson and the staus. The mass matrix of the charged scalars is given by:

$$
L_{m}=-\left[H_{d}^{-}, H_{u}^{-}, \tilde{\tau}_{L}^{-}, \tilde{\tau}_{R}^{-}\right] M_{S^{ \pm}}^{2}\left[\begin{array}{c}
H_{d}^{+} \\
H_{u}^{+} \\
\tilde{\tau}_{L}^{+} \\
\tilde{\tau}_{R}^{+}
\end{array}\right]+\text {h.c. } .
$$

For convenience we divide this $4 \times 4$ matrix into $2 \times 2$ blocks in the following way:

$$
M_{S^{ \pm}}^{2}=\left[\begin{array}{cc}
M_{H H}^{2} & M_{H \tilde{\tau}}^{2 T} \\
M_{H \tilde{\tau}}^{2} & M_{\tilde{\tau} \tilde{\tau}}^{2}
\end{array}\right]
$$

The charged Higgs block is given by

$$
\begin{gathered}
M_{H H}^{2}= \\
{\left[\begin{array}{cc}
B \mu \frac{v_{u}}{v_{d}}+\frac{1}{4} g^{2}\left(v_{u}^{2}-v_{3}^{2}\right)+\mu \epsilon_{3} \frac{v_{3}}{v_{d}}+\frac{1}{2} h_{\tau}^{2} v_{3}^{2}+\frac{t_{1}}{v_{d}} & B \mu+\frac{1}{4} g^{2} v_{d} v_{u} \\
B \mu+\frac{1}{4} g^{2} v_{d} v_{u} & B \mu \frac{v_{d}}{v_{u}}+\frac{1}{4} g^{2}\left(v_{d}^{2}+v_{3}^{2}\right)-B_{2} \epsilon_{3} \frac{v_{3}}{v_{u}}+\frac{t_{2}}{v_{u}}
\end{array}\right]}
\end{gathered}
$$

and the stau block is given by

$$
\begin{gathered}
M_{\tilde{\tau} \tilde{\tau}}^{2}= \\
{\left[\begin{array}{cc}
\frac{1}{2} h_{\tau}^{2} v_{d}^{2}-\frac{1}{4} g^{2}\left(v_{d}^{2}-v_{u}^{2}\right)+\mu \epsilon_{3} \frac{v_{d}}{v_{3}}-B_{2} \epsilon_{3} \frac{v_{u}}{v_{3}}+\frac{t_{3}}{v_{3}} & \frac{1}{\sqrt{2}} h_{\tau}\left(A_{\tau} v_{d}-\mu v_{u}\right) \\
\frac{1}{\sqrt{2}} h_{\tau}\left(A_{\tau} v_{d}-\mu v_{u}\right) & m_{E_{3}}^{2}+\frac{1}{2} h_{\tau}^{2}\left(v_{d}^{2}+v_{3}^{2}\right)-\frac{1}{4} g^{\prime 2}\left(v_{d}^{2}-v_{u}^{2}+v_{3}^{2}\right)
\end{array}\right]}
\end{gathered}
$$


We recover the usual stau and Higgs-mass matrices in the limit $v_{3}=\epsilon_{3}=0$ (we need to replace the expression of the third tadpole in Eq. (5) before taking the limit). The mixing between the charged Higgs sector and the stau sector is given by the following $2 \times 2$ block:

$$
M_{H \tilde{\tau}}^{2}=\left[\begin{array}{cc}
-\mu \epsilon_{3}-\frac{1}{2} h_{\tau}^{2} v_{d} v_{3}+\frac{1}{4} g^{2} v_{d} v_{3} & -B_{2} \epsilon_{3}+\frac{1}{4} g^{2} v_{u} v_{3} \\
-\frac{1}{\sqrt{2}} h_{\tau}\left(\epsilon_{3} v_{u}+A_{\tau} v_{3}\right) & -\frac{1}{\sqrt{2}} h_{\tau}\left(\mu v_{3}+\epsilon_{3} v_{d}\right)
\end{array}\right]
$$

and as expected, this mixing vanishes in the limit $v_{3}=\epsilon_{3}=0$. The charged scalar mass matrix in Eq. (16), after setting $t_{1}=t_{2}=t_{3}=0$, has determinant equal to zero, since one of the eigenvectors corresponds to the charged Goldstone boson "eaten" by the $\mathrm{W}$ boson. The mass matrices in Eqs. (16) are diagonalized by a rotation matrix $R_{S^{ \pm}}$:

$$
\left[\begin{array}{c}
G^{+} \\
H^{+} \\
\tilde{\tau}_{1}^{+} \\
\tilde{\tau}_{2}^{+}
\end{array}\right]=R_{S^{ \pm}}\left[\begin{array}{c}
H_{d}^{+} \\
H_{u}^{+} \\
\tilde{\tau}_{L}^{+} \\
\tilde{\tau}_{R}^{+}
\end{array}\right] .
$$

and the eigenvalues $\operatorname{are} \operatorname{diag}\left(0, \mathrm{~m}_{\mathrm{H}^{ \pm}}^{2}, \mathrm{~m}_{\tilde{\tau}_{1}^{ \pm}}^{2}, \mathrm{~m}_{\tilde{\tau}_{2}^{ \pm}}^{2}\right)=\mathrm{R}_{\mathrm{S}^{ \pm}} \mathrm{M}_{\mathrm{S}^{ \pm}}^{2} \mathrm{R}_{\mathrm{S}^{ \pm}}^{\mathrm{T}}$.

A similar mixing occur between the neutral Higgs bosons and the real part of the tau-sneutrino and between the pseudoscalar Higgs and the imaginary part of the tausneutrino [3, 5]. We denote the resulting scalar (pseudoscalar) state by $S_{i}^{0}\left(P_{i}^{0}\right)$ with $m_{S_{i}^{0}}<m_{S_{j}^{0}}$ for $i<j$.

\section{R-parity Violating Top Decays}

One of the major successes of Tevatron has been the discovery of the top-quark [14]. The large top mass implies a relatively small production cross section at the Tevatron. As a result the sum of all branching ratios of the top decays except $t \rightarrow W^{+} b$ is only restricted to be smaller than approximately $25 \%$ [15]. In the MSSM the top can decay according to: $t \rightarrow W^{+} b, t \rightarrow H^{+} b, t \rightarrow \tilde{\chi}_{1}^{0} \tilde{t}_{1}$, and $t \rightarrow \tilde{\chi}_{1}^{+} \tilde{b}_{1}$ (for their discussion in the MSSM see e.g. [16, 17 and references therein). The last mode is only listed for completeness, because it is practically ruled out by existing chargino and squark searches at LEP2 [18]. In the BRPV model additional decay modes occur:

$$
\begin{aligned}
t & \rightarrow \tilde{\tau}_{1}^{+} b \\
t & \rightarrow \tau^{+} \tilde{b}_{1} \\
t & \rightarrow \nu_{\tau} \tilde{t}_{1}
\end{aligned}
$$

For the following discussion we have randomly chosen $\mathrm{O}\left(10^{4}\right)$ points in the SUSY parameter space imposing the relevant experimental constraints on SUSY searches. The MSSM bounds on sparticles are in general not directly applicable to broken Rparity models and a reanalysis of the LEP and Tevatron data is necessary in order to determine the corresponding bounds. In the particular case of charginos such a reanalysis has been made and it was found that the bound on the chargino mass in the BRPV model is essentially the same as in the MSSM [19]. For definiteness we have taken: $m_{\tilde{t}_{1}}, m_{\tilde{b}_{1}}>80 \mathrm{GeV}, m_{S_{1}^{0}}>70 \mathrm{GeV}, \min \left(m_{H^{+}}, m_{\tilde{\tau}_{1}}\right)>70 \mathrm{GeV}$, and $m_{\tilde{\chi}_{1}^{+}}>90 \mathrm{GeV}$. Moreover, we have imposed the $\nu_{\tau}$ mass constraint $m_{\nu_{\tau}}<18 \mathrm{MeV}$ [20]. 
Here, one should bear in mind that the heavy $\nu_{\tau}$ possibility is certainly allowed by the present Super Kamiokande data, as they may be accounted for either by conversions of $\nu_{\mu}$ to sterile neutrinos [21], flavour changing muon-neutrino interactions [22] or $\nu_{\mu}$ decay [23. On the other hand cosmological and astrophysical limits on $m_{\nu_{\tau}}$ are obviated in the presence of neutrino decay and annihilation channels involving majorons, present in the model with spontaneous breaking. For a review see ref. [24] and for recent references see e.g. [25]. Therefore, this is the only conservative limit one can really apply on the tau-neutrino mass. However, as we will see in the following discussion of Fig. 1, the R-parity violating branching ratios may be sizeable even with a small tau-neutrino mass.

As an illustrative example we show in Fig. 1 the branching ratio for $t \rightarrow \tilde{\tau}_{1}^{+} b$ as a function of the R-parity violating parameter $v_{3}$ for different values of $\tan \beta=v_{2} / v_{1}$. We have varied $-500 \mathrm{GeV}<\epsilon_{3}<0 \mathrm{GeV}$ and $0 \mathrm{GeV}<B_{2}<500 \mathrm{GeV}$. The remaining parameters have been fixed as: $M=180 \mathrm{GeV}, \mu=200 \mathrm{GeV}, M_{E_{3}}=285 \mathrm{GeV}$, $A_{\tau}=280 \mathrm{GeV}, M_{Q}=285 \mathrm{GeV}, M_{U}=180 \mathrm{GeV}, M_{D}=190 \mathrm{GeV}, A_{t}=320 \mathrm{GeV}$, $A_{b}=120 \mathrm{GeV}$, and $B=50 \mathrm{GeV}$. We have imposed the constraint $m_{\nu_{\tau}}<18 \mathrm{MeV}$ (a) and $m_{\nu_{\tau}}<1 \mathrm{eV}$ (b). One immediately observes the following: (i) The larger $\tan \beta$ the larger is the branching ratio. (ii) The bound on $m_{\nu_{\tau}}$ does not lead to smaller branching ratios but to smaller allowed values of $v_{3}$ for fixed $\epsilon_{3}$. The first fact is understood in the following way: The stau mixes mainly with $H_{d}$ (since $\tilde{\tau}_{L}$ and $H_{d}$ have the same gauge quantum numbers). There is a strong mixing between the stau and the charged Higgs for the points where the branching ratio is above $\sim 10 \%$. Moreover, the coupling $H_{d} t b$ is proportional to $h_{b}$ which grows with $\tan \beta$. For the second point note that $m_{\nu_{\tau}}$ is proportional to $\epsilon_{3} v_{1}+\mu v_{3}$ [4]. Moreover, one can keep in principal $m_{\nu_{\tau}}$ fixed and one is still able to change the partial width $\Gamma\left(t \rightarrow \tilde{\tau}_{1}^{+} b\right)$ by varying $B_{2}$ and therefore the Higgs-Stau mixing.

In Fig. 2 we show the branching ratios $\operatorname{BR}\left(t \rightarrow \tau^{+} \tilde{b}_{1}\right)$ as a function of $\epsilon_{3}$ and $v_{3}$ for $\tan \beta=35$ and the other parameters as above (in the first case we have taken $\left.0<v_{3}<25 \mathrm{GeV}\right) . m_{\tilde{b}_{1}}$ grows with decreasing $\tan \beta$ if one keeps the other parameters fixed and therefore this decay mode will be kinematically forbidden for small $\tan \beta$. In Fig. 2a we see a strong correlation between $\epsilon_{3}$ and $\operatorname{BR}\left(t \rightarrow \tau^{+} \tilde{b}_{1}\right)$. This can be understood in the following way: in the chargino mass matrix (Eq. (đ)) the mixing between the leptons and the charginos disappears if one does the following rotation of the superfields: $\widehat{H}_{d} \rightarrow N\left(\mu \widehat{H}_{d}-\epsilon_{3} \widehat{L}_{3}\right)$ and $\widehat{L}_{3} \rightarrow N\left(\mu \widehat{L}_{3}+\epsilon_{3} \widehat{H}_{d}\right)$ (N being the normalization). In this basis the coupling between $t, \tau$, and $\tilde{b}_{1}$ is proportional $N h_{b} \epsilon_{3}$ leading to this feature. In Fig. 2 $\mathrm{b}$ we show the same branching ratio as a function of $v_{3}$. The fact that the branching ratio is not larger than $\sim 10 \%$ is a consequence of the limit on $m_{\nu_{\tau}}$. The parameters $v_{3}$ and $\epsilon_{3}$ are responsible for the mixing between the charginos and the tau-lepton. This mixing is the reason for the band observed in the figure.

In Fig. 3 we show the sum of all branching ratios for non-standard decay modes of the top quark as a function of $\epsilon_{3}(\mathrm{a})$ and $v_{3}(\mathrm{~b})$. One can see that existing Tevatron data already exclude parts of the parameter space which is not excluded by other data. In both cases we have cascade decays: 

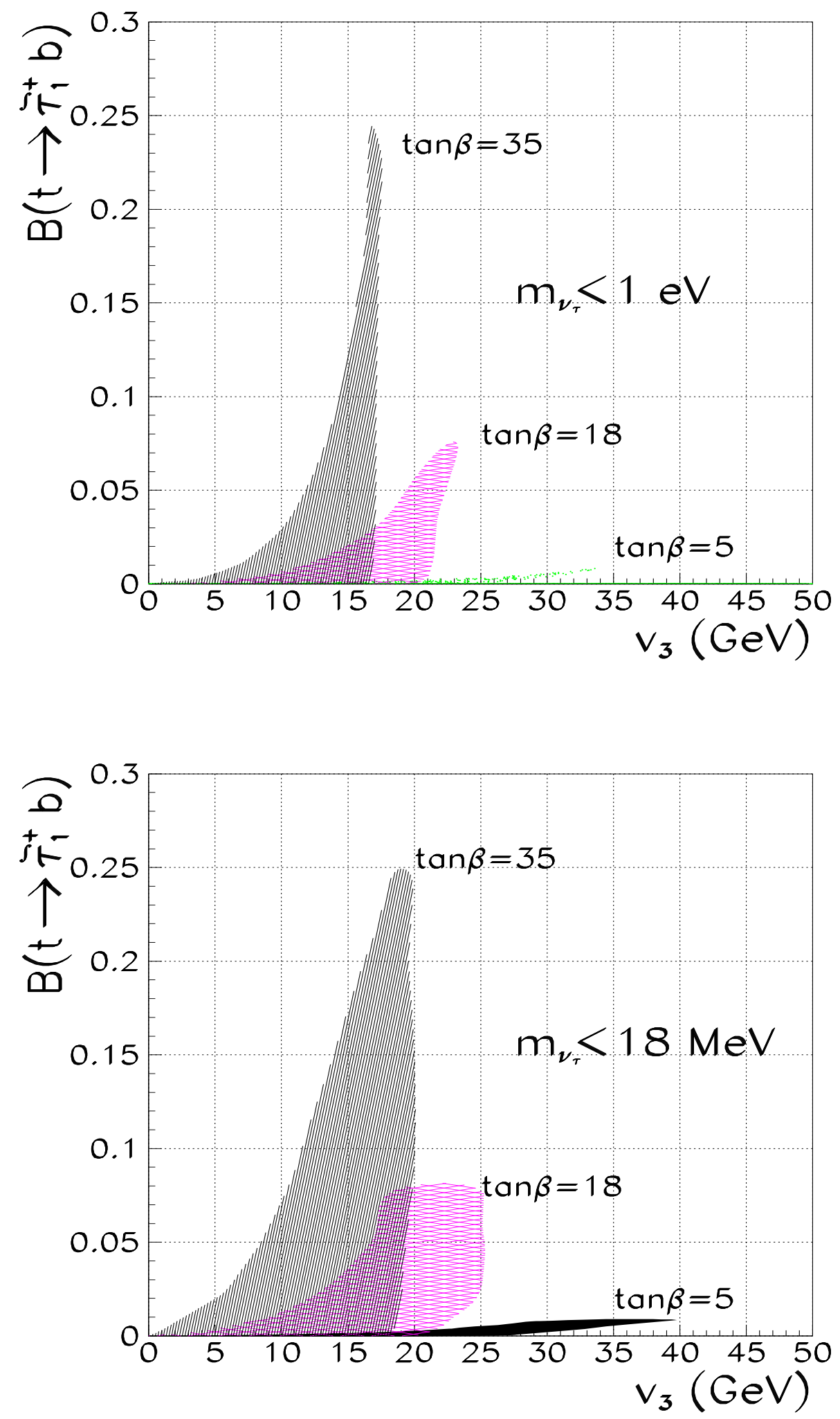

Figure 1: Branching Ratio $\operatorname{BR}\left(t \rightarrow \tilde{\tau}_{1}^{+} b\right)$ as a function of $v_{3}$ for different values of $\tan \beta$. The other parameters are given in the text. We have imposed the constraint $m_{\nu_{\tau}}<18 \mathrm{MeV}$ (a) and $m_{\nu_{\tau}}<1 \mathrm{eV}$ (b). 
a)

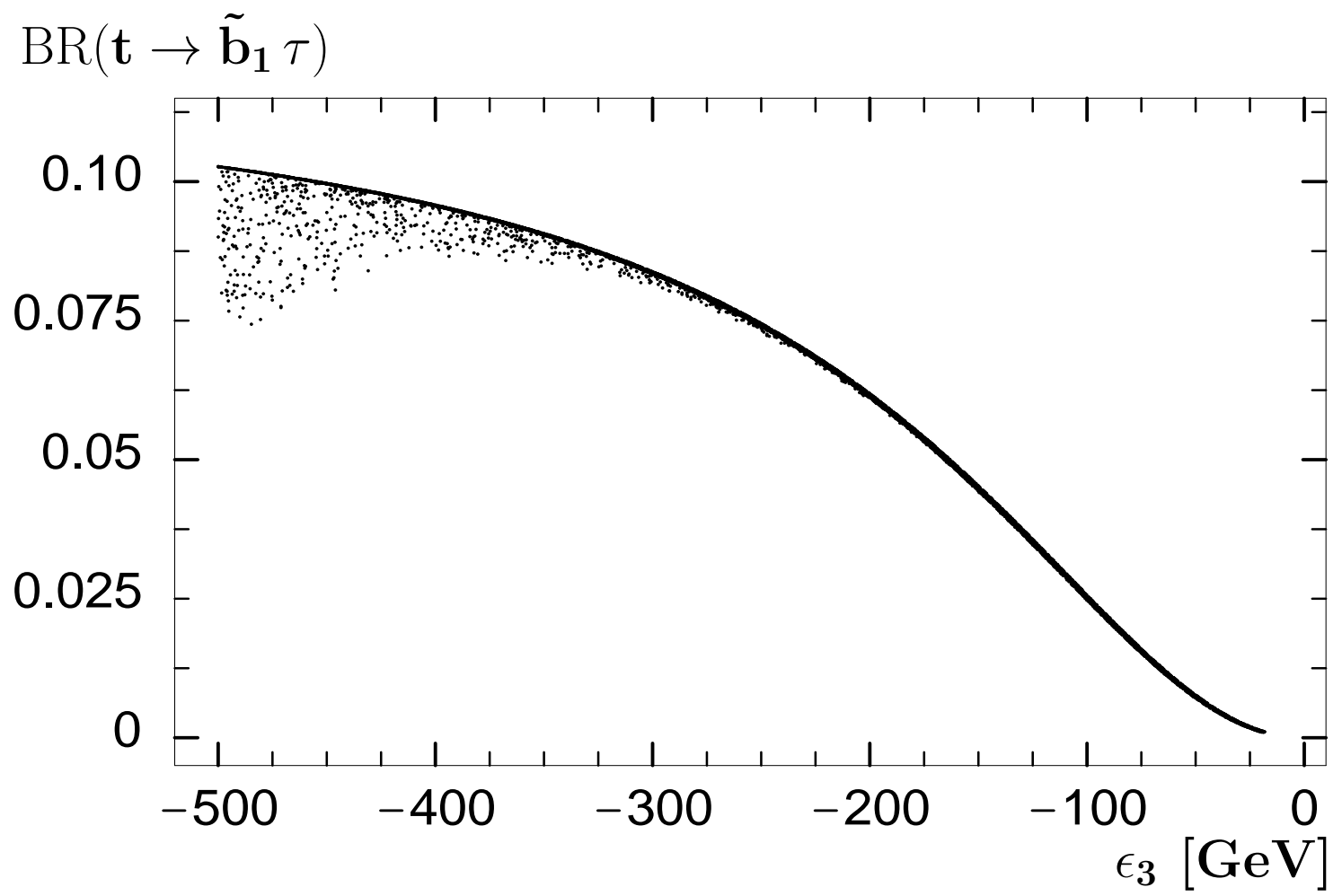

b)

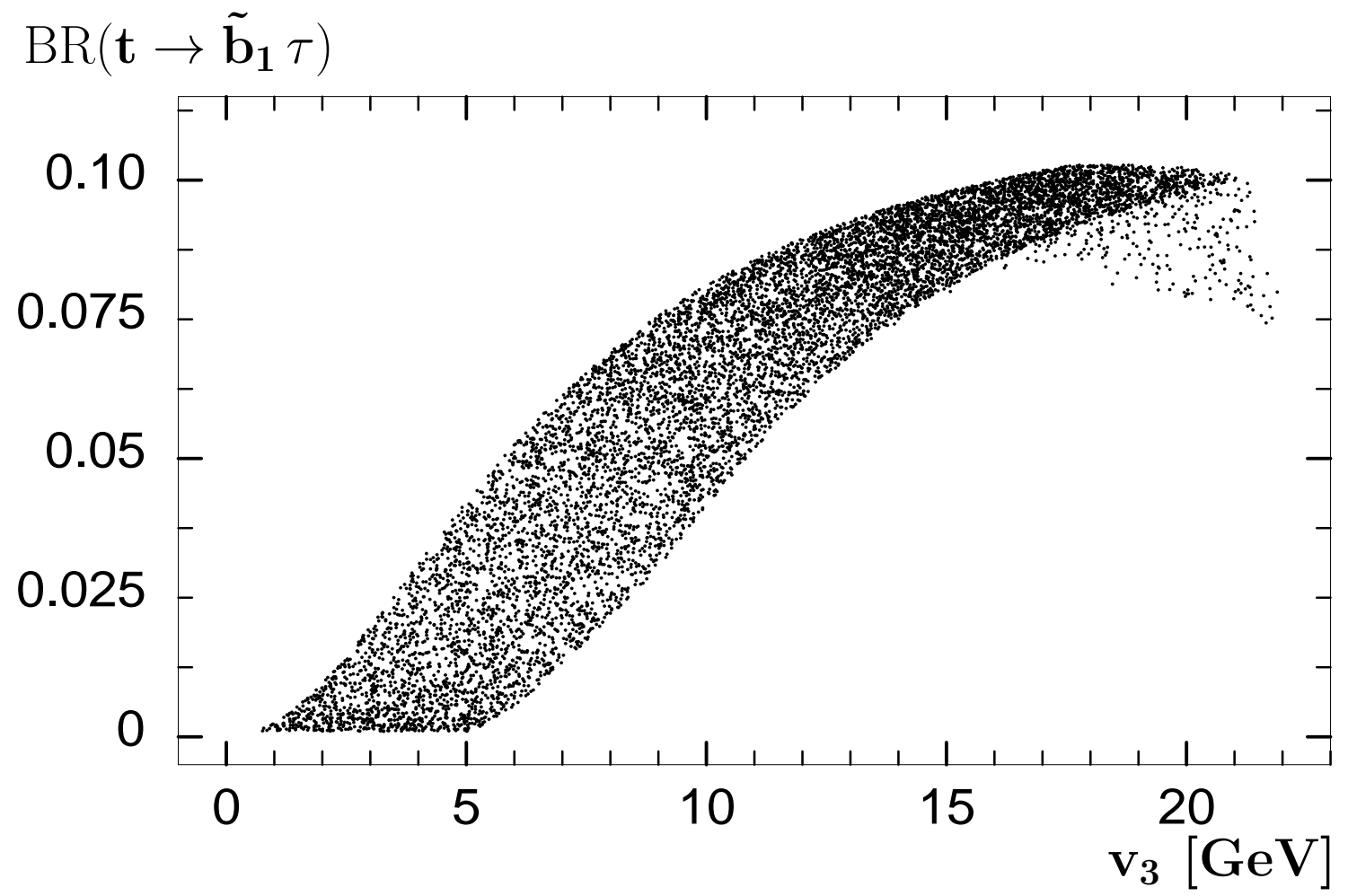

Figure 2: Branching Ratios for $t \rightarrow \tilde{b}_{1} \tau^{+}$as a function of the R-parity violating parameters $\epsilon_{3}$ (a) and $v_{3}$ (b). Here we have assumed $m_{\nu_{\tau}}<18 \mathrm{MeV}$ and the other parameters are given in the text. 
a)

$\mathbf{1}-\mathrm{BR}(\mathbf{t} \rightarrow \mathbf{W} \mathbf{b})$

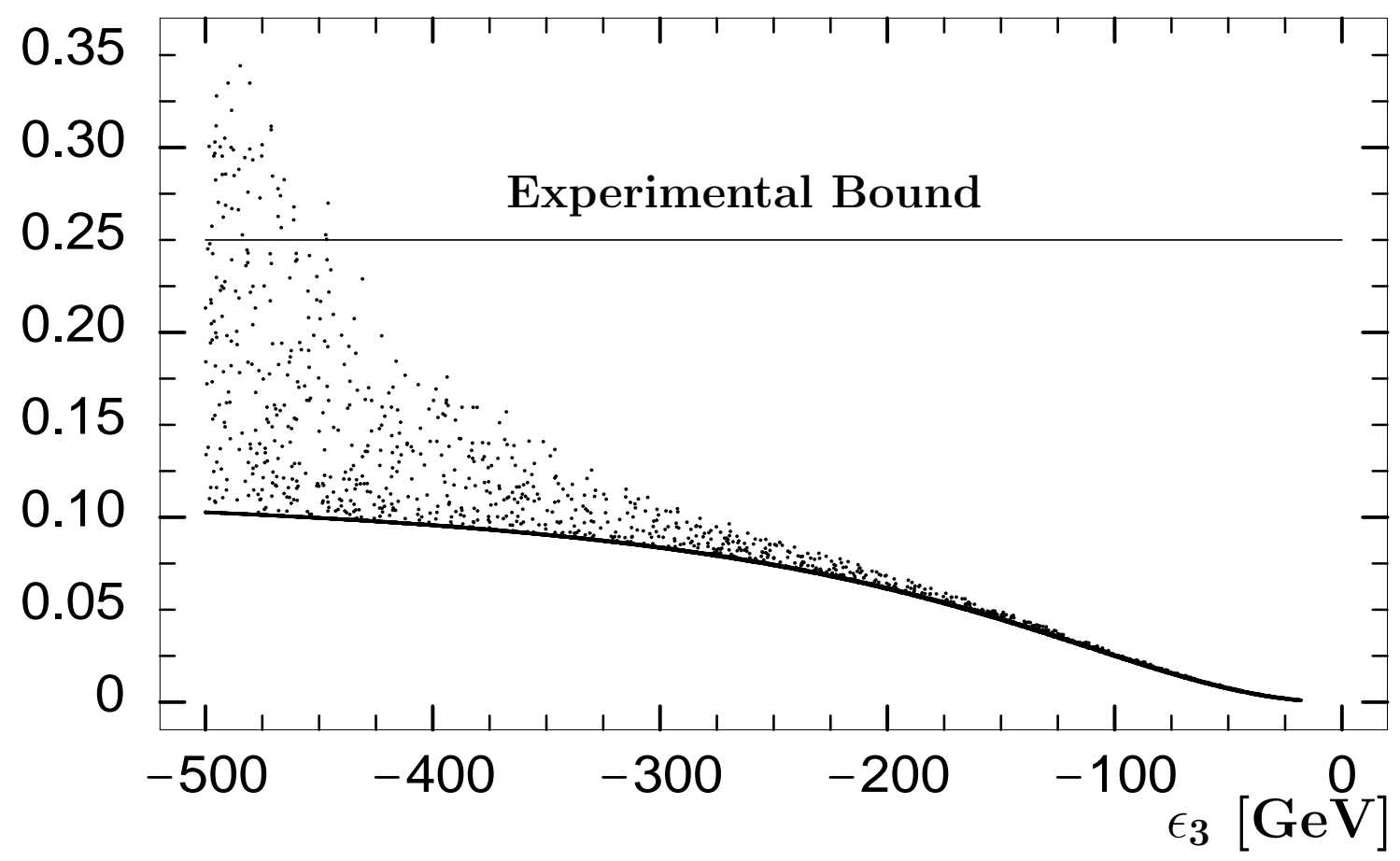

b)

$\mathbf{1}-\mathrm{BR}(\mathbf{t} \rightarrow \mathbf{W} \mathbf{b})$

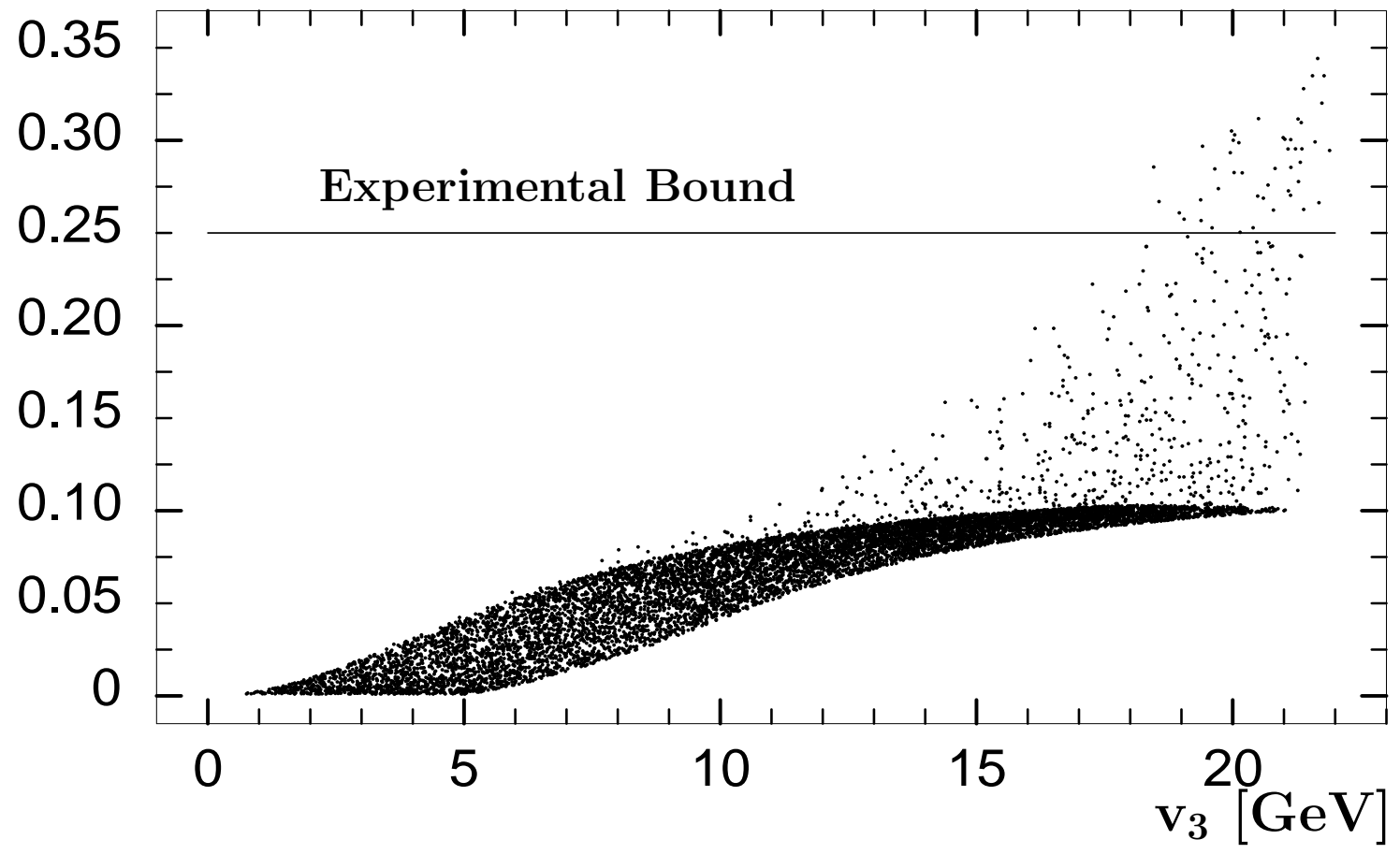

Figure 3: Sum of all branching Ratios different from $t \rightarrow W b$ as a function of $\epsilon_{3}$ (a) and $v_{3}(\mathbf{b})$. The parameters are chosen as in Fig. 2 . 


$$
\begin{aligned}
& t \rightarrow \tilde{\tau}_{1}^{+} b \rightarrow \tau^{+} \nu_{\tau} b \\
& \rightarrow \tau^{+} \tilde{\chi}_{1}^{0} b \rightarrow \tau^{+} f \bar{f} \nu_{\tau} b \\
& \rightarrow \tau^{+} f \bar{f}^{\prime} \tau^{ \pm} b \\
& \rightarrow \nu_{\tau} \tilde{\chi}_{1}^{+} b \rightarrow \nu_{\tau} f \bar{f}^{\prime} \nu_{\tau} b \\
& \rightarrow \nu_{\tau} f \bar{f} \tau^{+} b \\
& \rightarrow c s b \\
& t \rightarrow \tau^{+} \tilde{b}_{1} \rightarrow \tau^{+} \nu_{\tau} b \\
& \rightarrow \tau^{+} \tilde{\chi}_{1}^{0} b \rightarrow \tau^{+} f \bar{f} \nu_{\tau} b \\
& \rightarrow \tau^{+} f \bar{f}^{\prime} \tau^{ \pm} b
\end{aligned}
$$

In most cases there are two $\tau$-leptons and two $b$-quarks in the final state plus the possibility of additional leptons and/or jets. Therefore, $b$-tagging and a good $\tau$ identification are important for extracting these final states. Moreover, there is in general a large multiplicity of charged particles in the final state which should be helpful in reducing the background. The background stems mainly from the production of one or two gauge bosons plus additional jets. The conclusion in similar cases [26] has been that within its next run Tevatron will be sensitive for branching ratio measurements up to $10^{-3}-10^{-2}$ depending on the mode. Therefore, the observation of one the additional decay modes at the run 2 of Tevatron will give a strong hint on the underlying R-parity violating parameters.

We have performed a similar scan for small $\tan \beta(\leq 5)$. Here we have found that the non-standard decay modes are smaller: $1-\mathrm{BR}(t \rightarrow W b)<0.02$. This happens because their decay widths are in both cases proportional to the bottom Yukawa coupling squared. In case of $t \rightarrow \tilde{\tau}_{1}^{+} b$ this can be already seen from Fig. 1 and in case of $t \rightarrow \tau^{+} \tilde{b}_{1}$ is clear from the discussion of Fig. 2. Up to now we have not discussed the decay $t \rightarrow \nu_{\tau} \tilde{t}_{1}$ because it turns out that the branching ratio for this mode is tiny in the parameter region studied. The reason is that the Higgsino $\tilde{H}_{u}$ hardly mixes with $\nu_{\tau}$. Moreover, the coupling is proportional to $m_{\nu_{\tau}} / m_{W}$.

\section{Conclusions}

We have seen that the R-parity violating decay modes of the top quark $t \rightarrow \tilde{\tau}_{1}^{+} b$ and $t \rightarrow \tilde{b}_{1} \tau^{+}$can have large branching ratios, especially if the tau neutrino is heavy. We have also shown that existing Tevatron data already probe the theoretical parameters, and the prospects for further improvement at the Run 2 of the Tevatron are promising. Finally we have also verified that the magnitude of the R-parity violating top decay branching ratios considered here remains large even when the $\nu_{\tau}$ mass becomes very small, as perhaps indicated by the recent atmospheric neutrino data.

\section{Acknowledgements}

This work was supported by DGICYT under grant number PB95-1077, by the TMR network grant ERBFMRXCT960090 the European Union and by Accion Integrada Hispano-Austriaca. L. N. was supported by CSIC. W. P. was supported by the "Fonds zur Förderung der wissenschaftlichen Forschung" of Austria, project No. P13139-PHY. 


\section{References}

[1] H.E. Haber and G.L. Kane, Phys. Rep. 117, 75 (1985).

[2] C.S. Aulakh, R.N. Mohapatra, Phys. Lett. 119B, 136 (1982); L. Hall and M. Suzuki, Nucl. Phys. B231, 419 (1984); G.G. Ross, J.W.F. Valle, Phys. Lett. 151B 375 (1985); J. Ellis, G. Gelmini, C. Jarlskog, G.G. Ross, J.W.F. Valle, Phys. Lett. 150B 142 (1985).

[3] M.A. Díaz, J.C. Romão, and J.W.F. Valle, Nucl. Phys. B524 23 (1998).

[4] M.A. Díaz, hep-ph/9711435, hep-ph/9712213; J.C. Romão, hep-ph/9712362; J.W.F. Valle, talk at PASCOS 98, hep-ph/9808292.

[5] F. de Campos, M.A. García-Jareño, A.S. Joshipura, J. Rosiek, and J.W.F. Valle, Nucl. Phys. B451, 3 (1995); A. Akeroyd, M.A. Díaz, J. Ferrandis, M.A. GarcíaJareño, J.W.F. Valle, Nucl. Phys. B529 (1998) 3-22 hep-ph/9707395; For early papers see [2].

[6] A.S. Joshipura and M. Nowakowski, Phys. Rev. D 51, 2421 (1995); T. Banks, Y. Grossman, E. Nardi, and Y. Nir, Phys. Rev. D 52, 5319 (1995); F. Vissani and A.Yu. Smirnov, Nucl. Phys. B460, 37 (1996); R. Hempfling, Nucl. Phys. B478, 3 (1996); F.M. Borzumati, Y. Grossman, E. Nardi, Y. Nir, Phys. Lett. B 384, 123 (1996); H.P. Nilles and N. Polonsky, Nucl. Phys. B484, 33 (1997); B. de Carlos, P.L. White, Phys. Rev. D 55, 4222 (1997); E. Nardi, Phys. Rev. D 55, 5772 (1997); S. Roy and B. Mukhopadhyaya, Phys. Rev. D 55, 7020 (1997); A. Faessler, S. Kovalenko, F. Simkovic, hep-ph/9712535; M. Carena, S. Pokorski, and C.E.M. Wagner, hep-ph/9801251; M.E. Gómez and K. Tamvakis, hep-ph/9801348.

[7] A. Masiero and J.W.F. Valle, Phys. Lett. B251, 273 (1990); J.C. Romão, A. Ioannissyan and J.W.F. Valle, Phys. Rev. D55, 427 (1997).

[8] M.A. Díaz, J. Ferrandis, J.C. Romão, and J.W.F. Valle, Phys. Lett. Bxxx, xxx (1999) hep-ph/9801391.

[9] For reviews see J.W.F. Valle, hep-ph/9712277 and hep-ph/9603307.

[10] V. Berezinskii, A.S. Joshipura, J.W.F. Valle. Phys.Rev.D57, 147-151 (1998).

[11] Hall \& Suzuki in ref. [2].

[12] H. Dreiner, R.J.N. Phillips, Nucl. Phys. B367 591-613,1991; V. Barger, M.S. Berger, R.J.N. Phillips, T. Wöhrmann. Phys. Rev. D 53 ,6407 (1996); J. Erler, J. Feng, and N. Polonsky, Phys. Rev. Lett. 78, 3063-3066 (1997).

[13] A. Akeroyd, M.A. Díaz, J. Ferrandis, M.A. Garcia-Jareño and J.W.F. Valle, Nucl. Phys. B529 3 (1998), (hep-ph/9797395).

[14] CDF Coll., F. Abe et al., Phys. Rev. Lett. 74, 2626 (1995); D0 Coll., S. Abachi et al., Phys. Rev. Lett. 74, 2422 (1995); Phys. Rev. Lett. 74, 2632 (1995); Phys. Rev. D52, 4877 (1995).

[15] S. Mrenna and C.-P. Yuan, Phys. Lett. B367 188 (1996); M. Hosch et al., Phys. Rev. D58:034002 (1998); G.V. Velev (for the CDF Coll.) FERMILAB-Conf98/192-E. 
[16] K. Hidaka, Y. Kizukuri and T. Kon, Phys. Lett. B278, 155 (1992); M. Drees et al., Phys. Rev. D54, 5598 (1996); M. Hosch et al., Phys. Rev. D58, 034002 (1998); D.A. Dicus, D.J. Muller and S. Nandi, Phys. Rev. D59, 055007 (1999).

[17] F.M. Borzumati and N. Polonsky, hep-ph/9602433, Proc. of the workshop on ' $e^{+} e^{-}$collisions at TeV energies: The Physics Potential', DESY 96-123D, p. 41, ed. P.M. Zerwas.

[18] R. Rebecchi, talk given at ICHEP98, Vancouver, Canada, July 23-29, 1998.

[19] F. de Campos, O.J.P. Eboli, M.A. Garcia-Jareno, J.W.F. Valle, hep-ph 9710545, Nucl. Phys. B546, (1999) 33-51

[20] I. Nikolic, Nucl. Phys. B, Proc. Suppl. 66, 214 (1998).

[21] M.C. Gonzalez-Garcia, H. Nunokawa, O.L.G. Peres, J.W.F. Valle, Nucl. Phys. B543 (1999) 3 hep-ph 9807305].

[22] M.C. Gonzalez-Garcia et al, hep-ph 9809531, Phys. Rev. Lett., in press.

[23] M.C. Gonzalez-Garcia et al, in preparation.

[24] J.W.F. Valle, in Prog. Part. Nucl. Phys. 26:91-171,1991 and [4].

[25] A. D. Dolgov, S. Pastor, J.C. Romao, J.W.F. Valle, Nucl. Phys. B496 (1997) 2440, hep-ph 9610507, A.D. Dolgov, S.H. Hansen, S. Pastor, D.V. Semikoz, hep-ph 9809598 .

[26] T. LeCompte, Rare Decays Working Group Summary, workshop on top-quark physics at Run II, Fermi National Accelerator Laboratory, October 16 - 18, 1998. 